\title{
Variación ontogénica y sexual de Oecomys bicolor (Rodentia, Cricetidae) de Perú y Ecuador
}

\section{Ontogenetic and sexual variation of Oecomys bicolor (Rodentia, Cricetidae) from Peru and Ecuador}

\section{María Fernanda Menajovsky ${ }^{1 *}$, Víctor Pacheco ${ }^{1,2}$}

\author{
1 Universidad Nacional Mayor de San Marcos, Museo de Historia Natural, Departamento de Mastozoología, Apartado 14-0434. Lima 14, Perú. \\ 2 Instituto de Ciencias Biológicas “Antonio Raimondi”, Facultad de Ciencias Biológicas, Universidad Nacional Mayor de San Marcos, Lima, Perú. \\ *Autor para correspondencia \\ Email María Fernanda Menajovsky: mafe.menajovsky@gmail.com \\ Email Víctor Pacheco: vpachecot@unmsm.edu.pe
}

\begin{abstract}
Resumen
Oecomys es un género rico en diversidad de especies de tamaño pequeño a mediano. Dentro del género, la especie $O$. bicolor es reconocida morfológicamente con relativa facilidad; sin embargo, se ha reportado en distintas poblaciones de la especie variaciones individuales,-con respecto al tamaño y color, por lo que podría tratarse de un complejo de especies. La información intraespecífica es muy poca a pesar de su amplia distribución; no obstante, esta información es necesaria para la delimitación de la especie, así como explicar variaciones en poblaciones. Por ello, en el presente estudio se analizaron 148 especímenes de O. bicolor, con el objetivo de evaluar la variación no geográfica referida al sexo y edad. Se analizaron 13 variables cráneo-dentales. Mediante aproximaciones estadísticas univariadas y multivariadas se demostró que el dimorfismo sexual no es significativo; por otro lado, la variación ontogénica es considerable entre jóvenes y adultos, estos resultados son consistentes con estudios previos realizados en roedores sigmodontinos, Se recomienda continuar los estudios de variación geográfica tomando en cuenta las edades 3,4 y 5, y agrupando machos y hembras.
\end{abstract}

Palabras clave: Orizomyini; Ontogenia; Dimorfismo Sexual, Morfometría geométrica,

\section{Abstract}

Oecomys is a moderately specious genus of small to medium size. Within the genus, the species $O$. bicolor is readily recognized; however, it has been reported individual variation in size and color from different populations suggesting that the species could be a species complex. Intraspecific information in O. bicolor is limited despite its wide distribution; but, this information is necessary for delimiting the species as well as explaining variations within populations. Therefore, in the present study 148 specimens of $O$. bicolor were analyzed, with the objective of evaluating the non-geographic variation of the species related to sex and age. 13 skull-dental variables were analyzed. Univariate and multivariate statistical approximations showed that sexual dimorphism is not significant; on the other hand, the ontogenic variation is considerable among young adults, these results are consistent with previous studies in sigmodontine rodents. It is recommended to continue studies of geographic variation with only ages 3,4 and 5, and treating males and females collectively.

Keywords: Oryzomyini; Ontogeny; Sexual dimorphism; Geometric morphometrics

Citación:

Menajovsky M.F., V. Pacheco. 2017. Variación ontogénica y sexual de Oecomys bicolor (Rodentia, Cricetidae) de Perú y Ecuador. Revista peruana de biología 24(4): 383 - 390 (diciembre 2017). doi: http://dx.doi. org/10.15381/rpb.v24i4.13918

$\begin{array}{ll}\text { Presentado: } & 25 / 10 / 2017 \\ \text { Aceptado: } & 29 / 11 / 2017\end{array}$

Publicado online: $20 / 12 / 2017$
Fuentes de financiamiento:

Nº96-2014-FONDECYT-DE; Inter-American Institute for Global Change Research (IAI) CRN3076; US National Science Foundation (Grant GE0-1128040); Travel Grants of World Wildlife Fund Peru: TD10-TD16, TD96-TD99, TE01 y TE02.

Información sobre los autores:

MFM se encargó de la obtención, análisis de datos y redacción del presente trabajo. VP confirmó la identificación de las muestras; realizó análisis de datos obtenidos así como apoyó en el diseño y la revisión del presente trabajo.

Los autores declaran que no existen conflictos de intereses al presentar este trabajo de investigación. 


\section{Introducción}

Los ratones arrozaleros del género Oecomys Thomas 1906, son pequeños roedores de la tribu Oryzomyini (15-90 gramos; Carleton \& Musser 2015), que se caracterizan por presentar una piel suave, patas cortas y anchas con dedos relativamente largos y cola unicoloreada, moderadamente más larga que la cabeza y el cuerpo (Voss \& Carleton 1993, Weksler 2006, Carleton \& Musser 2015). Este género tiene hábitos principalmente arborícolas y su distribución abarca zonas tropicales y subtropicales de Centro América y Sudamérica (Musser \& Carleton 2005, Carleton et al. 2009). Habitan principalmente bosques lluviosos de tierras bajas tropicales, donde la mayor diversidad de especies se concentra en la cuenca del río Amazonas (Musser \& Carleton 2005, Carleton \& Musser 2015). El género es rico en diversidad de especies y actualmente contiene 16 taxones válidos (Musser \& Carleton 2005, Carleton et al. 2009, Carleton \& Musser 2015).

Oecomys bicolor es una especie considerada monotípica y reconocida morfológicamente por su tamaño pequeño y patrón de coloración dorso-ventral, con el vientre blanco y el dorso pardo o pardo rojizo (Patton et al. 2000, Carleton \& Musser 2015). Su distribución es amplia, ocurriendo el este de Panamá al oeste de Ecuador y desde el oeste de Colombia y norte y centro de Venezuela, hasta la Amazonia peruana y ecuatoriana, además de extenderse por Bolivia y el sur central de Brasil (Carleton \& Musser 2015). Sin embargo, se ha reportado que esta especie presenta variaciones en tamaño, color y en secuencias de ADN mitocondrial, lo cual requiere una investigación adicional para poder afirmar si corresponden con variaciones intraespecíficas o especies candidatas adicionales (Patton et al. 2000, Carleton \& Musser 2015), lo cual amerita un estudio de variabilidad intraespecífica para entender los efectos de edad y sexo sobre la variabilidad geográfica a nivel morfológico.

Diversas publicaciones han tratado algunos aspectos morfológicos, cariotípicos, ecológicos, reproductivos y moleculares de Oecomys bicolor (e. g., Patton et al. 2000, Voss et al. 2001, Carleton \& Musser 2015); sin embargo, la información no incluye la variación intraespecífica (Mayr 1969, Weksler et al. 2006), con excepción de Patton et al. (2000), quienes evaluaron datos morfométricos obtenidos para $O$. bicolor de la región del río Jurua, Brasil, encontrando ausencia significativa de dimorfismo sexual en sus resultados de variación no geográfica.

El presente estudio tiene por objetivo realizar un análisis de la variación no geográfica de $O$. bicolor presentes en la amazonia occidental de Perú y Ecuador, analizando la ontogenía y el dimorfismo sexual mediante métodos estadísticos para conocer la variabilidad existente en la especie que no se encuentra relacionada a factores geográficos para posteriores estudios.

\section{Material y métodos}

Muestras examinadas.- Se revisaron 148 especímenes de Oecomys bicolor de ocho departamentos de Perú y siete provincias de Ecuador (Figura 1); se incluyó36 individuos de los 148 especímenes de $O$. bicolor, dichos individuos pertenecientes al departamento de Madre de Dios para el análisis correspondiente utilizando una sola zona geográfica, la cual contaba con la mayor cantidad de individuos pertenecientes a todas las clases de edades y ambos sexos para realizar una comparación posterior con resultados globales y analizar el posible efecto geográfico.
Los especímenes empleados se encuentran en los siguientes museos: Museo de Historia Natural de la Universidad Nacional mayor de San Marcos (MUSM), Lima, Perú; Museo de Historia Natural Gustavo Orcés - Escuela Politécnica Nacional (EPN), Quito, Ecuador; Museo de Zoología (QCAZ) - Pontificia Universidad Católica de Ecuador, Quito, Ecuador y el Instituto Nacional de Biodiversidad, Quito, Ecuador (MECN). Las localidades exactas de colectas con sus respectivos datos de ubicación se encuentran disponible en el Apéndice 1.

Dimorfismo sexual y clasificación de edades.- Los individuos fueron agrupados por sexo (hembras y machos) y clases de edad. Las clases de edad fueron determinadas con base al desarrollo de los molares y el desgaste de sus superficies, además de características del pelaje. Las edades consideradas fueron: Clase de edad 1: primer y segundo molar conflexo labial abierto y conspicuo; tercer molar no erupcionado o recién erupcionado, con las cúspides cerradas, sin exposición de dentina; pelaje dorsal más oscuro y pelaje ventral grisáceo; clase de edad 2: primer y segundo molar con las cúspides principales (¿cuáles?) no desgastadas y exposición de dentina, algunos flexos cerrados; tercer molar medianamente a moderadamente desgastado; pelaje dorsal más oscuro y pelaje ventral grisáceo; clase de edad 3: primer y segundo molar con cúspides moderadamente erosionadas y alta exposición de dentina y algunas fosas molares presentes; tercer molar presenta marcado desgaste siendo la superficie casi plana

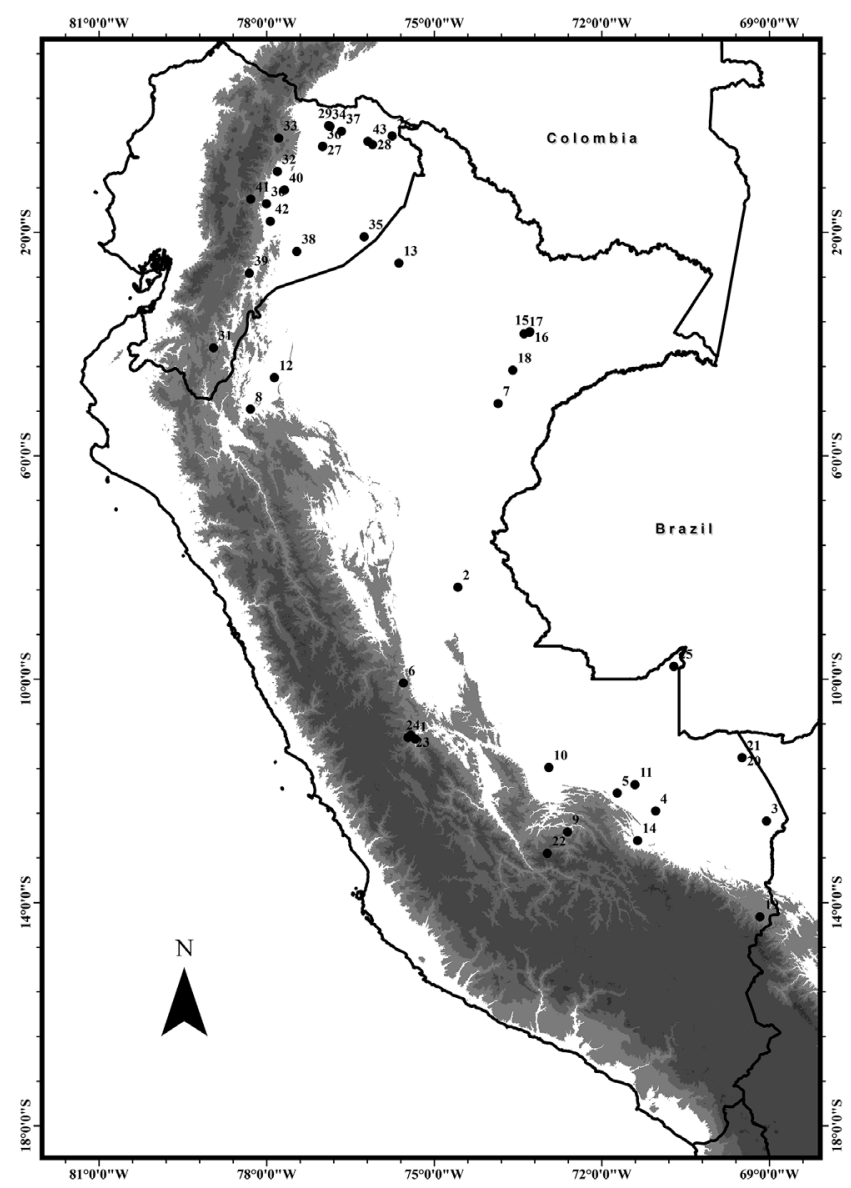

Figura 1. Registros de localidades para Oecomys bicolor analizadas en el presente estudio. Los números indican los nombres de cada localidad. La franja más oscura representa la elevación de los Andes (Apéndice 1). 
o plana; pelaje dorsal marrón rojizo y pelaje ventral crema; clase de edad 4: primer y segundo molar con desgaste mayor, cúspides planas y exposición mayor de dentina, presencia de fosas molares; tercer molar plano, con exposición de dentina; pelaje dorsal marrón rojizo y pelaje ventral crema; clase de edad 5: los tres molares con completo desgaste; alta exposición de dentina y erosión de fosas molares; pelaje dorsal marrón rojizo y pelaje ventral crema (Voss1991, Percequillo1998).

Medidas cráneo-dentales.- Se seleccionaron 13 medidas cráneo-dentales (expresadas en milímetros [mm]) (Figura 2) y las mismas fueron realizadas con un vernier de $0.01 \mathrm{~mm}$ de precisión: Longitud total del cráneo (LTC): desde el margen anterior de los nasales hasta la parte posterior del hueso occipital. Longitud cóndilo- incisivo (LCI): desde la curvatura mayor del incisivo superior hasta la superficie del cóndilo occipital, del mismo lado del cráneo. Longitud diastema (LD): desde la corona del primer molar superior hasta el lado interno del incisivo superior, del mismo lado del cráneo. Longitud foramen incisivo
(LFI): la longitud mayor, medida desde el borde anterior hasta el posterior del foramen. Ancho del foramen incisivo (AFI): la amplitud mayor medida entre los márgenes laterales del foramen. Ancho del paladar (AP): medida en la parte interna del paladar, a la altura del primer molar. Longitud de nasales (LN): desde la parte anterior del nasal hasta la sutura naso-frontal. Ancho mínimo entre las orbitas (AMO): la distancia más corta a través de los frontales en la fosa orbital. Ancho de los arcos cigomáticos (AC): mayor distancia entre los arcos cigomáticos, cercana a las raíces del escamoso, medida a través del cráneo. Ancho de las bulas auditoria (AB): medida desde la sutura petrosa hasta el proceso del ectotímpano. Longitud orbital (LO): dimensión mayor de la fosa orbital, entre el escamoso y las raíces maxilares del arco cigomático. Longitud fosa mesopterigoidea (LFM): longitud mayor medida desde el borde anterior hasta el posterior de la fosa. Ancho de la fosa mesopterigoidea (AFM): la amplitud mayor medida entre las márgenes laterales de la fosa (Weksler 2016).

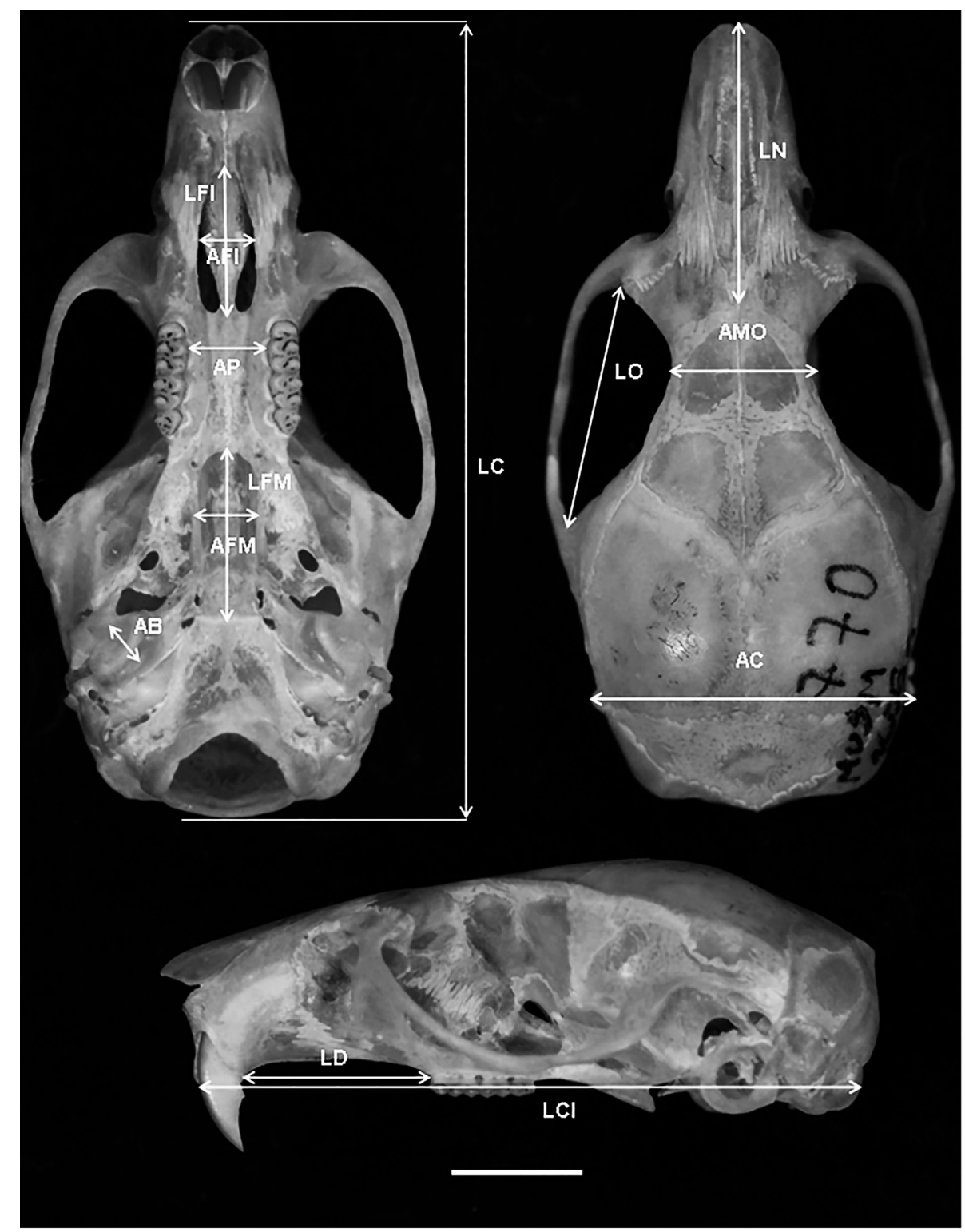

Figura 2. Vistas dorsal, ventral y lateral del cráneo de un individuo adulto de Oecomys bicolor (MUSM 16253), ilustrando las 13 medidas seleccionadas para este estudio. Escala: $5 \mathrm{~mm}$. Abreviaciones definidas en el texto. 
Análisis estadísticos.- Se utilizó la prueba de KolmogorovSmirnov (KS) para evaluar la normalidad univariada y Shapiro Wilk (SW) para la normalidad multivariada con una significancia de $\mathrm{p}<0.05$. Se aplicó la prueba $\mathrm{T}$ de Student para verificar el efecto del dimorfismo sexual en las variables empleadas. Para evaluar el efecto de la edad en la muestra se realizó un análisis multivariado de varianza (MANOVA) con Traza de Pillai y ANOVA con Tukey's post hoc para los siguientes análisis: variación de edad con machos y hembras agrupados, luego variación de edad con sexos separados, comparando cada clase de edad independientemente. Los análisis estadísticos se realizaron en el programa R 3.4.1(R Core Team 2017).

\section{Resultados}

Variación intraespecífica según sexo y edad.- La prueba univariada de normalidad Kolmogorov-Smirnov , mostró una distribución normal para todas las variables empleadas, al igual que la prueba de Shapiro Wilk con respecto a la distribución normal multivariada (resultados no mostrados).

Los resultados de la prueba T de Student para los individuos de Madre de Dios, así como la muestra global de Perú y Ecuador sugieren que no existe variación significativa en ninguno de los caracteres que se deba al dimorfismo sexual, los resultados muestran un valor de significancia $\mathrm{p}>0.4$ para todos los caracteres, incluso algunos cercanos a $\mathrm{p}=1$.

En los resultados del MANOVA para evaluar la variación debida a la edad en la población de Madre de Dios (Tabla 1), se pudo observar que las variables LCI, LFI, AFI, AP, LN, LO y LFM muestran diferencias significativas $(\mathrm{p}<0.05)$. La prueba ANOVA encontró variación significativa de los caracteres evaluados principalmente en los individuos de Clase de edad 1, comparando las clases de edad por pares (Tabla 2). Los resultados significativos se muestran en negrita en las tablas respectivas.

Cabe resaltar que la prueba de ANOVA muestra que existen diferencias significativas al evaluar la variable AP para los pares de edades $2 / 5,3 / 5$ y $4 / 5$ (Tabla 2).
Los resultados del MANOVA para evaluar la variación respecto a la edad en la muestra global de Perú y Ecuador sugieren que las variables LC, LCI, LD, LFI, LN, AMO, LO y LFM presentan diferencias significativas $(\mathrm{p}<0.05)$ (Tabla 3).

Los resultados del ANOVA comparando las clases de edad por pares mostraron que los individuos de la clase de edad 1 presentan diferencias significativas en las mismas variables LC, LCI, LD, LFI, LN, AMO, LO y LFM al compararlos con las subsiguientes clases de edad; existe también una diferencia significativa en la variable AMO relacionada con la clase de edad 2.

Tabla 1. Resultados de la Prueba de MANOVA con Traza de Pillai producto de la evaluación del efecto de la edad en la especie O. bicolor de la localidad Madre de Dios, Perú, utilizando 13 mediciones cráneo dentales. Var= variables cráneo-dental. $\mathrm{F}=$ Estadístico $\mathrm{F}$. El significado de las abreviaturas para las mediciones se explican en el texto. Se muestra en negrita los valores significativos ( $p<0.05)$, obtenidos en la prueba.

\begin{tabular}{ccc}
\hline & MANOVA & Pillai \\
\cline { 2 - 3 } Var & F & \\
\hline LC & 2.234 & 0.089 \\
LCI & 3.703 & $\mathbf{0 . 0 1 4}$ \\
LD & 1.661 & 0.186 \\
LFI & 2.815 & $\mathbf{0 . 0 4 3}$ \\
AFI & 3.011 & $\mathbf{0 . 0 3 4}$ \\
AP & 3.839 & $\mathbf{0 . 0 1 2}$ \\
LN & 3.498 & $\mathbf{0 . 0 1 9}$ \\
AMO & 0.676 & 0.614 \\
AC & 1.365 & 0.27 \\
AB & 1.563 & 0.211 \\
LO & 2.683 & $\mathbf{0 . 0 5 1}$ \\
LFM & 3.054 & $\mathbf{0 . 0 3 2}$ \\
AFM & 0.773 & 0.552 \\
\hline
\end{tabular}

Tabla 2. Resultados de la prueba de ANOVA con análisis Tukey's Post Hoc para la comparación de lasclases de edad en la especie Oecomys bicolor de la localidad Madre de Dios, Perú, utilizando 13 mediciones cráneo dentales. Var= variable cráneodental. F= Estadístico F. El significado de las abreviaturas para las mediciones se explican en el texto. Se muestra en negrita los valores significativos $(p<0.05)$, obtenidos en la prueba.

\begin{tabular}{ccccccccccccc}
\hline & \multicolumn{1}{c}{ ANOVA } & \multicolumn{10}{c}{ Tukey } \\
\cline { 2 - 11 } Var & $\mathbf{F}$ & $\mathbf{1 / 2}$ & $\mathbf{1 / 3}$ & $\mathbf{1 / 4}$ & $\mathbf{1 / 5}$ & $\mathbf{2 / 3}$ & $\mathbf{2 / 4}$ & $\mathbf{2 / 5}$ & $\mathbf{3} / \mathbf{4}$ & $\mathbf{3} / \mathbf{5}$ & $\mathbf{4} / \mathbf{5}$ \\
\hline LC & 2.234 & $\mathbf{0 . 0 5 9}$ & $\mathbf{0 . 0 5 8}$ & 0.112 & $\mathbf{0 . 0 5 5}$ & 0.998 & 0.964 & 0.996 & 0.987 & 0.968 & 0.895 \\
LCI & 3.703 & $\mathbf{0 . 0 3 5}$ & $\mathbf{0 . 0 1 5}$ & $\mathbf{0 . 0 0 5}$ & $\mathbf{0 . 0 1 6}$ & 0.989 & 0.629 & 0.905 & 0.725 & 0.969 & 0.999 \\
LD & 1.661 & 0.839 & 0.722 & 0.498 & 0.230 & 0.996 & 0.796 & 0.321 & 0.856 & 0.335 & 0.809 \\
LFI & 2.815 & 0.189 & 0.326 & 0.507 & $\mathbf{0 . 0 4 4}$ & 0.870 & 0.687 & 0.618 & 0.972 & 0.166 & 0.118 \\
AFI & 3.011 & 0.092 & 0.118 & 0.088 & $\mathbf{0 . 0 1 5}$ & 0.979 & 1.000 & 0.515 & 0.978 & 0.204 & 0.482 \\
AP & 3.839 & 0.478 & 0.274 & 0.425 & $\mathbf{0 . 0 1 4}$ & 0.960 & 0.999 & $\mathbf{0 . 0 3 2}$ & 0.983 & $\mathbf{0 . 0 4 1}$ & $\mathbf{0 . 0 3 5}$ \\
LN & 3.498 & $\mathbf{0 . 0 4 4}$ & $\mathbf{0 . 0 5 1}$ & $\mathbf{0 . 0 6 0}$ & $\mathbf{0 . 0 0 6}$ & 0.990 & 0.997 & 0.495 & 0.999 & 0.220 & 0.330 \\
AMO & 0.676 & 0.993 & 0.999 & 1.000 & 0.997 & 0.595 & 0.987 & 0.755 & 0.883 & 0.999 & 0.922 \\
AC & 1.365 & 0.160 & 0.275 & 0.245 & 0.424 & 0.88 & 0.985 & 0.943 & 0.997 & 1.000 & 0.997 \\
AB & 1.563 & 0.736 & 0.901 & 1.000 & 0.983 & 0.900 & 0.173 & 0.852 & 0.326 & 0.990 & 0.915 \\
LO & 2.683 & 0.791 & 0.365 & 0.232 & 0.097 & 0.594 & 0.323 & 0.111 & 0.909 & 0.411 & 0.830 \\
LFM & 3.054 & $\mathbf{0 . 0 3 7}$ & $\mathbf{0 . 0 3 5}$ & $\mathbf{0 . 0 2 3}$ & $\mathbf{0 . 0 1 4}$ & 0.998 & 0.997 & 0.855 & 0.953 & 0.676 & 0.942 \\
AFM & 0.773 & 0.945 & 0.798 & 0.609 & 0.935 & 0.948 & 0.677 & 1.000 & 0.903 & 0.994 & 0.882 \\
\hline
\end{tabular}


Los resultados significativos se muestran en negrita en las tablas respectivas (Tabla 4).

Al emplear la misma prueba estadística tomando en cuenta la separación por sexo de los individuos, tanto para la muestra de Madre de Dios como para la muestra global de Perú y Ecuador, los resultados evidencian consistencia; las variables diferenciadas se mantienen sin importar la discriminación según dimorfismo sexual de los individuos de $O$. bicolor.

Tabla 3. Resultados de la prueba MANOVA con Traza de Pillai producto de la evaluación del efecto de la edad en la especie O. bicolor de la amazonia occidental de Perú y Ecuador, utilizando 13 mediciones cráneo-dentales. Var= variable cráneo- dental. $\mathrm{F}=$ Estadístico $\mathrm{F}$. El significado de las abreviaturas para las mediciones se explican en el texto. Se muestra en negrita los valores significativos obtenidos en la prueba $\mathrm{p}<0.05$

\begin{tabular}{ccc} 
& MANOVA & Pillai \\
\cline { 2 - 3 } Var & F & \\
\hline LC & 13.321 & $\mathbf{0 . 0 0 0 3}$ \\
LCI & 15.933 & $\mathbf{0 . 0 0 0 1}$ \\
LD & 17.619 & $\mathbf{4 . 6 9 E}-05$ \\
LFI & 6.310 & $\mathbf{0 . 0 1 3}$ \\
AFI & 2.741 & 0.099 \\
AP & 0.101 & 0.750 \\
LN & 23.811 & $\mathbf{2 . 7 7 E - 0 6}$ \\
AMO & 21.279 & $\mathbf{8 . 6 6 E}-06$ \\
AC & 0.423 & 0.516 \\
AB & 3.594 & 0.059 \\
LO & 18.847 & $\mathbf{2 . 6 4 E}-05$ \\
LFM & 20.258 & $\mathbf{1 . 3 8 E - 0 5}$ \\
AFM & 2.995 & 0.085 \\
\hline
\end{tabular}

\section{Discusión}

En el presente estudio se demostró que existe variación ontogénica en la población de Madre de Dios (Perú) de O. bicolor (principalmente aquellos de la clase de edad 1), sugiriéndose que los individuos más jóvenes son los más distintos en la muestra. Por otro lado, el dimorfismo sexual no mostró un efecto significativo en la población.

Los resultados también revelaron que las variables de longitud total del cráneo y del rostro son las más variantes con respecto a la edad de los individuos; estas variables se ven reflejadas en las medidas cráneo- dentales: LCI, LFI, LN, LO y LFM, las cuales se esperarían incrementen de acuerdo con el tamańo corporal y edad del individuo, determinando la expansión del rostro y dentadura durante el periodo postnatal (Prado \& Percequillo 2011).

La medida cráneo- dental AP es la única variable que exhibe diferencias en $O$. bicolor en más de una clase de edad, dicha observación se realizó al evaluar la población de Madre de Dios, debido a que esto se puede ver asociado a cambios específicos de la población o estar influenciado por el tamaño de muestra del análisis, no se tomó en cuenta al momento de hacer conclusiones de la población de $O$. bicolor presente en Perú y Ecuador en el estudio.

$\mathrm{Al}$ realizar el análisis global en individuos de la amazonia occidental de Perú y Ecuador tres caracteres tomaron significancia en las pruebas estadísticas (LC, LD y AMO); se puede sugerir que dichos caracteres si estarían relacionados a una variabilidad geográfica, al solo ser encontrados significativos en la muestra global de $O$. bicolor, ya que esta compararía diferentes áreas geográficas de la especie.

Así mismo, se encontraron diferencias en algunas variables de la muestra global con respecto a la clase de edad 2; por lo cual se podría sospechar de formas no adultas en dicha clases de edad y apoyaría resultados anteriores (Jiménez et al. 2013, Prado \& Percequillo 2011, Patton et al. 2000). El análisis global también reveló una ausencia de dimorfismo sexual en la especie según lo esperado.

Tabla 4. Resultados de la prueba de ANOVA con análisis Tukey's Post Hoc producto de la comparación de las clases de edad en la especie Oecomys bicolor de la amazonia occidental de Perú y Ecuador, utilizando 13 mediciones cráneo-dentales. Var= variable cráneo- dental. F= Estadístico F. El significado de las abreviaturas para las mediciones se explican en el texto. Se muestra en negrita los valores significativos $(p<0.05)$, obtenidos en la prueba.

\begin{tabular}{|c|c|c|c|c|c|c|c|c|c|c|c|}
\hline \multirow[b]{2}{*}{ Var } & \multicolumn{6}{|l|}{ ANOVA } & \multicolumn{5}{|c|}{ key } \\
\hline & $\mathbf{F}$ & $1 / 2$ & $1 / 3$ & $1 / 4$ & $1 / 5$ & $2 / 3$ & $2 / 4$ & $2 / 5$ & $3 / 4$ & $3 / 5$ & $4 / 5$ \\
\hline $\mathrm{LC}$ & 8.541 & 0.000 & 0.000 & 0.000 & 0.004 & 0.997 & 0.819 & 0.999 & 0.895 & 0.999 & 0.988 \\
\hline LCI & 8.732 & 0.000 & 0.000 & 0.000 & 0.024 & 0.996 & 0.236 & 0.999 & 0.289 & 0.997 & 0.612 \\
\hline $\mathrm{LD}$ & 10.79 & 0.000 & 0.000 & 0.000 & 0.000 & 0.999 & 0.413 & 0.949 & 0.418 & 0.964 & 0.994 \\
\hline LFI & 6.048 & 0.000 & 0.000 & 0.002 & 0.002 & 0.939 & 0.991 & 0.960 & 0.999 & 0.817 & 0.893 \\
\hline AFI & 3.781 & 0.006 & 0.002 & 0.010 & 0.256 & 0.999 & 0.997 & 0.996 & 0.999 & 0.991 & 0.983 \\
\hline $\mathrm{AP}$ & 0.801 & 0.602 & 0.562 & 0.464 & 0.983 & 1.000 & 0.988 & 0.986 & 0.982 & 0.986 & 0.937 \\
\hline $\mathrm{LN}$ & 10.941 & 0.000 & 0.000 & 0.000 & 0.000 & 0.999 & 0.869 & 0.194 & 0.890 & 0.194 & 0.607 \\
\hline AMO & 4.521 & 0.976 & 0.292 & 0.242 & 0.006 & 0.230 & 0.258 & 0.004 & 0.978 & 0.071 & 0.256 \\
\hline $\mathrm{AC}$ & 11.562 & 0.000 & 0.000 & 0.000 & 0.076 & 0.852 & 0.958 & 0.250 & 0.999 & 0.491 & 0.582 \\
\hline $\mathrm{AB}$ & 1.641 & 0.986 & 0.998 & 0.697 & 0.965 & 0.996 & 0.154 & 0.780 & 0.194 & 0.852 & 0.996 \\
\hline LO & 8.155 & 0.000 & 0.000 & 0.000 & 0.000 & 0.999 & 0.296 & 0.727 & 0.319 & 0.774 & 0.999 \\
\hline LFM & 5.929 & 0.009 & 0.000 & 0.000 & 0.020 & 0.890 & 0.190 & 0.875 & 0.470 & 0.983 & 0.989 \\
\hline AFM & 1.973 & 0.219 & 0.067 & 0.142 & 0.878 & 0.963 & 0.976 & 0.979 & 0.999 & 0.893 & 0.900 \\
\hline
\end{tabular}


En ambos análisis, tanto para la población de Madre de Dios, así como el estudio global en Perú y Ecuador podemos observar que la edad más distinta seria la clase de edad 1, tomando en cuenta las diferencias en la clase de edad 2 para algunas variables. Los individuos de clase de edad 3 en adelante son los mejores candidatos para el análisis de adultos en las muestras.

Dichos individuos en esa clase son similares entre sí y podrían ser utilizados en estudios geográficos de la especie sin presentar mayor variación entre los individuos de la misma población. No existe otro estudio en roedores sigmodontinos que afirme que los individuos se podrían considerar adultos a partir de la clase de edad 2, pero sí a partir de la clase 3 (Patton et al. 2000, Prado y Percequillo 2011), los cuales también están incluidos en los resultados del estudio. Por lo tanto al tener un número considerable de muestra sería recomendable utilizar individuos a partir de la clase de edad 3 en adelante.

La diferenciación ontogénica en $O$. bicolor ya se había reportado en individuos de la Amazonia brasilera por Patton et al. (2000), los autores analizaron 22 individuos de la especie en diversas localidades adyacentes al río Juruá, encontrando diferencias en casi todas las medidas externas y cráneo - dentales utilizadas (24 variables en total) respecto a las clases de edades y ninguna diferenciación debido al dimorfismo sexual de la especie; Nuestros resultados concuerdan plenamente.

Patton et al. (2000) realizaron también un análisis similar para las población de Oecomys roberti presentes en río Juruá, encontrando resultados similares al analizar la variabilidad por sexo y edad, documentando ausencia de dimorfismo sexual y diferencias significativas por edad. Los autores concluyeron, y nuestros resultados concuerdan, que se debe considerar el factor de edad en estudios para todas las especies del género Oecomys en diversas localidades.

Así mismo dicha diferenciación se puede encontrar en diversos estudios; Jiménez et al. (2013), analizaron más de 100 individuos de Akodon orophilus de los departamentos Amazonas, San Martin y Huánuco en Perú, utilizando 19 medidas cráneo - dentales y análisis de MANOVA para comparar las distintas clases de edades y ambos sexos; los autores encontraron que las clases de edad 4 y 5 eran las únicas que no presentaron diferencias significativas a nivel de las variables analizadas.

En el estudio de Aegialomys xanthaeolus realizado por Prado \& Percequillo (2011), se analizaron más de 400 especímenes en 90 localidades distintas transandinas de Perú y Ecuador, los especimenes fueron separados de acuerdo a las localidades y se evaluaron 19 medida cráneo-dentales. Los autores encontraron una variación significativa en relación a las clases de edades (al menos 12 variables muestran diferenciación), demostraron que las clases de edad 1 y 2 son las más distintas y consideradas juveniles, también hallaron diferencias en edades adultas, concluyeron que las edades 4 y 5 serían las más similares entre sí.

Un punto importante en el estudio de Prado \& Percequillo (2011) es que sí se obtuvo una fuerte diferenciación debida al dimorfismo sexual en la clase de edad 3, sin embargo, la ausencia de dimorfismo sexual es el patrón común en roedores de diversas familias (Carleton \& Musser 1989), como se ha visto en la especie Dasymys incomtus (Mullin et al. 2001), algunos roedores cricétidos como Transandinomys talamancae (Musser\& Williams 1985), el género Microryzomys (Carleton \& Musser 1989) y el género Oryzomys (Musser et al. 1998), incluyendo a especies del género Oecomys (Patton et al. 2000) y el presente trabajo.

Se concluye que para las poblaciones de O. bicolor de Perú y Ecuador, el dimorfismo sexual no es un componente importante de variación no-geográfica; y que confirmándose la presencia de variación ontogenética se sugiere realizar estudios subsecuentes de variabilidad geográfica con ejemplares de una clase de edad mayor a 2, agrupando machos y hembras.

\section{Agradecimientos}

A Pamela Sánchez y Dennisse Ruelas del departamento de Mastozoología del Museo de Historia Natural (UNMSM), Lima, Perú por el apoyo en el procesamiento de datos y sugerencias para mejorar el manuscrito. A Pablo Moreno del Museo de Historia Natural Gustavo Orcés - Escuela Politécnica Nacional (EPN), Quito, Ecuador; a Santiago Burneo del Museo de Zoología QCAZ - Pontificia Universidad Católica de Ecuador, Quito, Ecuador y a Jorge Brito del Instituto Nacional de Biodiversidad, Quito, Ecuador por facilitar permisos y acceso a las respectivas colecciones.

Parte de los especímenes fueron obtenidos con los proyectos: "Caracterización molecular de roedores reservorios de enfermedades emergentes en la región amazónica y modelamiento de su distribución para la identificación de áreas de alto: caso hantavirus" con Convenio de Adjudicación Nº96-2014-FONDECYT-DE suscrito entre el Fondo Nacional de Desarrollo Científico, Tecnológico y de Innovación Tecnológica (FONDECYT - CONCYTEC) y la Universidad Nacional Mayor de San Marcos; Inter-American Institute for Global Change Research (IAI) CRN3076, financiado por US National Science Foundation (Grant GE0-1128040) y Travel Grants of World Wildlife Fund Peru: TD 10 - TD 16, TD 96-TD 99, TE 01 y TE 02.

\section{Literatura citada}

Carleton M.D., L.H. Emmons \& G.G. Musser. 2009. A new species of the rodent genus Oecomys (Cricetidae: Sigmodontinae; Oryzomyini) from eastern Bolivia, with emended definitions of O. concolor (Wagner) and O. mamorae (Thomas). American Museum Novitates 3661:1-32.doi: https://doi. org/10.1206/612.1

Carleton M.D. \& G.G. Musser.1984. Muroid Rodents. En: S. Anderson y J.K. Jones Jr. (Eds). Orders and families of recent mammals of the world. John Wiley Publications, New York. Pp. 289-379.

Carleton M. D., \& G. G. Musser. 1989. Systematic studies of oryzomyine rodents (Muridae, Sigmodontinae): a synopsis of Microryzomys. Bulletin of the American Museum of Natural History 191. Falta números de páginas

Carleton M.D. \& G.G. Musser. 2015. Genus Oecomys Thomas 1906. En J.L. Patton, U.F.J. Pardiñas, y G. D’Elía. (Eds). Mammals of South America. Volumen 2, Rodents. The University of Chicago Press, Chicago, IL. Pp. 395-416.

Jiménez, C.F., V. Pacheco \& D. Vivas.2013. An introduction to the systematics of Akodon orophilus Osgood, 1913 (Rodentia: Cricetidae) with the description of a new species. Zootaxa 3669 (3): 223-242.

Mullin S.K., N. Pillay \& P.J. Taylor. 2001. Non-geographic morphometric variation in the water rat Dasymys incomtus (Rodentia: Muridae) in southern Africa. Durban Museum Novitates 26:38-44.

Musser G.G, M.D. Carleton, E. Brothers \& A.L. Gardner. 1998. Systematic studies of Oryzomyine rodents (Muridae, Sigmodontinae): diagnoses and distributions of species formerly assigned to Oryzomys "capito". Bulletin of the American Museum of Natural History 236:1-376.

Musser G.G. \& M.D. Carleton. 2005. Superfamily Muroidea. En: D.E Wilson y D.M Reeder (Eds.). Mammals species of the 
World, a taxonomic and geographic reference. 3rd Edition, Vol. 2. The Johns Hopkins University Press, Baltimore. Pp. 894-1531.

Musser G. G. \& M.M. Williams. 1985. Systematic studies of oryzomyine rodents (Muridae): definitions of Oryzomys villosus and Oryzomys talamancae. American Museum Novitates 2810: $1-22$

Patton J.L., M.N.F Da Silva \& J.R. Malcolm. 2000. Mammals of the rio Juruá and the evolutionary and ecological diversification of Amazonia. Bulletin of the American Museum of Natural History 244:118-132.doi:https://doi.org/10.1206/00030090(2000)244<0001:MOTRJA>2.0.CO;2

Patton J. L. \& M.A. Rogers. 1983. Systematic implications of nongeographic variation in the spiny rat genus Proechimys (Echimyidae). Zeitschrift für Säugetierkunde 48(6):363370

Patton J. L., U.F. Pardiñas \& G.D.'Elía. 2015. Mammals of South America, volume 2: Rodents (Vol. 2). University of Chicago Press.

Percequillo A. R., E.Hingst-Zaher \& C.R. Bonvicino. 2008. Systematic review of genus Cerradomys Weksler, Percequillo and Voss, 2006 (Rodentia: Cricetidae: Sigmodontinae: Oryzomyini), with description of two new species from eastern Brazil. American Museum Novitates 3622:1-46.doi:https:// doi.org/10.1206/495.1

Percequillo A.R. 1998. Sistemática de Oryzomys Baird, 1858 do Leste do Brasil (Muroidea, Sigmodontinae). Tesis para optar grado de Doctor, Universidade de Sao Paulo.
Prado J. R. \& A. R. Percequillo. 2011. Ontogenetic and sexual variation in cranial characters of Aegialomys xanthaeolus (Thomas, 1894) (Cricetidae: Sigmodontinae) from Ecuador and Peru. Papéis Avulsos de Zoologia (São Paulo) 51(9): 155-177.

R Core Team (2017) R: A language and environment for statistical computing. R Foundation for Statistical Computing, Vienna, Austria. Available from: http://www.R-project.org/.

Voss R.S. \& M.D. Carleton. 1993. A new genus for Hesperomys molitor Winge and Holochilus magnus Hershkovitz (Mammalia, Muridae) with an analysis of its phylogenetic relationships. American Museum Novitates 3085:1-39.

Voss R.S. 1991. An introduction to the Neotropical muroid rodent genus Zygodontomys. Bulletin of the American Museum of Natural History 210: 1-113.

Voss, R.S., D.P. Lunde \& N.B. Simmons. 2001. The mammals of Paracou, French Guiana: a Neotropical lowland rainforest fauna Part 2. Non volant species. Bulletin of the American Museum of Natural History 263: 1-236.

Weksler M. 2006. Phylogenetic relationships of Oryzomyine rodents (Muroidea: Sigmodontinae): separate and combined analyses of morphological and molecular data. Bulletin of the American Museum of Natural History 296: 1-149.doi: https://doi.org/10.1206/0003-0090(2006)296[0001:PRO ORM]2.0.CO;2

Weksler M., A.R. Percequillo \& R.S. Voss. 2006. Ten new genera of Oryzomyine rodents (Cricetidae: Sigmodontinae). American MuseumNovitates 3537:1-29.doi: https://doi. org/10.1206/0003-0082(2006)3537[1:TNGOOR]2.0. $\mathrm{CO} ; 2$

Apéndice 1. Gacetilla de localidades. Especímenes empleados en los análisis y mapeados.

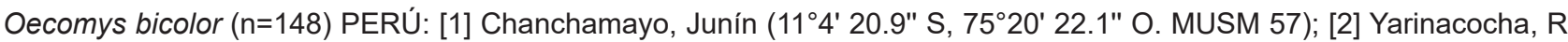
Ucayali ( $8^{\circ} 21^{\prime} 19.7^{\prime \prime} \mathrm{S}, 74^{\circ} 34^{\prime} 31.4^{\prime \prime}$ O. MUSM 58, 2535); [3] Reserva Cusco Amazónico, 15 km NE de Puerto Maldonado, Las Piedras, Madre de Dios (12 32' 26.8" S, 69 3' 9.5" O. MUSM 6288, 6290, 6291, 6292, 6293, 6294, 6295, 6296, 6297, 6298, 6299, 6300, 6301, 6302, 6303, 6304, 6305, 6306, 6307, 6308, 6309, 6310, 6312, 6313, 6314 , 6315 , 6317, 6318); [4] Hacienda Amazonia, R. Alto Madre de Dios, frente a Atalaya, Madre de Dios (12 $21^{\prime} 38.5^{\prime \prime}$ S, $71^{\circ} 2^{\prime}$ 8.4" O. MUSM 9215); [5] Río Manu, Parque Nacional del Manu, Puesto de Vigilancia de

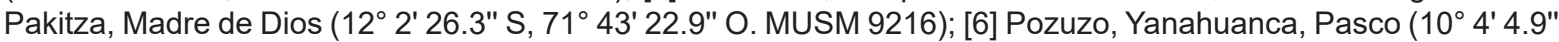
S, $75^{\circ} 33^{\prime}$ 6.1" O. MUSM 1269, 12696, 12697, 12700, 12701); [7] Requena, Yaquerana; Rio Galvez, San Juan, Yaquerana, Loreto (5 3' 49.3"S, $73^{\circ}$ 51' 22.9" O. MUSM 11208, 11211, 11213, 11215); [8] Bagua, Imaza, Imacita, Comunidad Aguaruna Yamayakat, Imaza, Amazonas (5 9' 50.8" S, 78 17' 18.6" O. MUSM 12046, 12047, 12053); [9] La Convención, Tangoshiari, Echarate, Cusco (12 44' 8.7" S, 72 36' 51.4" O. MUSM 13423); [10] La Convención,

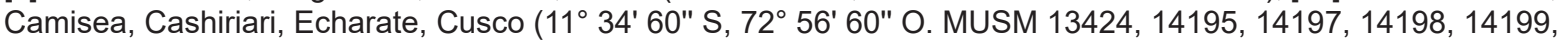
14200, 14201, 14202, 14203, 14204, 14209, 14210, 14216); [11] Manu, Estación Biológica Cocha Cashu, Madre

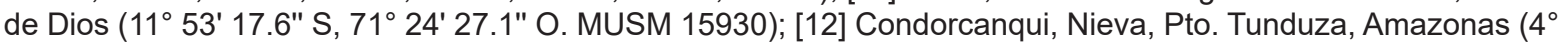
36' 3.6" S, $77^{\circ}$ 51' 39.7" O. MUSM 16249, 16251, 16252, 16253); [13] Rio Pucacuro, Collpa Salvador, margen derecha, Tigre, Loreto ( $2^{\circ} 33^{\prime} 0^{\prime \prime} \mathrm{S}, 7^{\circ} 37^{\prime}$ 60" O. MUSM 17606, 17651); [14] Consuelo, 15.9 km SW Pilcopata, Kosñipata, Cusco (12 $53^{\prime} 22.5^{\prime \prime} \mathrm{S}, 71^{\circ} 21^{\prime} 34.2^{\prime \prime}$ O. MUSM 19946); [15] Cahuide km 57 carretera lquitos-Nauta casa de Sra. Gonzales, San Juan Bautista, Loreto (3 47' 25.3" S, 73 17' 57.5" O. MUSM 33951, 33952, 33953); [16] El Triunfo Km 49.5 carretera Iquitos-Nauta, O del camino, San Juan Bautista, Loreto ( $3^{\circ} 46^{\prime} 33^{\prime \prime} \mathrm{S}, 7^{\circ} 17^{\prime}$ 21.5" O. MUSM 33954); [17] Mishana, río Nanay, banco sur, chacra de Eva, San Juan Bautista, Loreto $\left(3^{\circ} 48^{\prime}\right.$ 54.8" S, 73² 23' 26.7" O. MUSM 33959, 33960, 33961,33962); [18] Carretera Iquitos-Nauta, km 28.8, San Juan Bautista, Loreto (4 28' 3" S, 73 35' 38.3" O. MUSM 34903); [19] Yanahuaya, Puno (14 15' 27.5" S, 69 10' 9.5" O. MUSM 35087); [20] 1000m E de La Novia (ca. 8km al N del Caserío Mavila, sobre la carretera interoceánica),



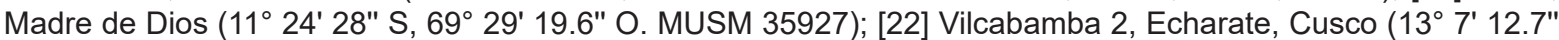

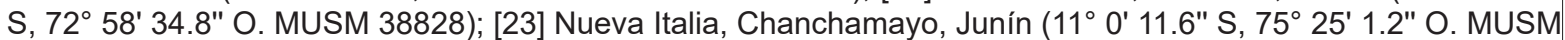

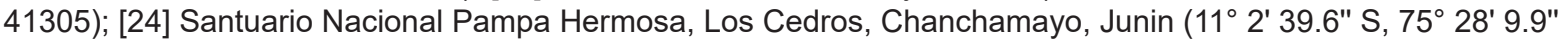


O. MUSM 41306, 41307); [25] Concesión de Conservación Río La Novia, Purús, Ucayali (946'17.7" S, $70^{\circ} 42^{\prime}$ 33.9" O. MUSM 44398, 44399, 44400, 44402, 44403, 44404, 44405, 44407, 44408, 44409, 44410, 44578, 44579, 44580, 44581, 44582). ECUADOR: [27] Ávila Viejo, Orellana (0²7' 16.3" S, 76 59' 42.1" O. PUCE 3422); [28] Pañacocha, Sucumbíos ( $0^{\circ} 25^{\prime} 27.9^{\prime \prime} \mathrm{S}, 76^{\circ} 6^{\prime}$ 9.7" O. PUCE 3738); [29] Comuna Yamanunka, Sucumbíos $\left(0^{\circ} 5^{\prime}\right.$ 20.1" N, 76 53' 23.1" O. PUCE 10145); [30] Área Operación AGIP - Bloque 10, Pastaza (1²9' 17.6" S, 78 $0^{\prime}$ 11.2" O. PUCE 9971, 10085); [31] El Kimi, Zamora Chinchipe (4 3' 56.1" S, $78^{\circ} 57^{\prime}$ 1.3" O. PUCE 15899); [32] Huamaní, Parroquia Cotundo, vía Hollín-Loreto, Archidona, Napo (0 54' 30.8" S, 77 48' 29.3" O. EPN 5937); [33] Las Cabeceras, Oriente, Quijos, Napo (0 18' 36.1" S, $77^{\circ} 47^{\prime} 1.8^{\prime \prime}$ O. EPN 5943); [34] Rio Aguarico, Lago Agrio, Sucumbíos (0 5' 60" N, 7652' 0" O. EPN 5950); [35] Rio Conambo, Pastaza (2 4' 39" S, 76 15' 15.9" O. EPN 5953); [36] Guiyero, Saladero km 34, vía Pompeya - Iro, Aguarico, Orellana (0²7' 40.9" S, 76 59' 57.6" O. EPN 10469, 10472, 10545); [37] Pañacocha, río Panayacu, Bosque Protector Pañacocha, Shushufindi, Sucumbíos $\left(0^{\circ}\right.$ 11' 10.1" S, $76^{\circ} 39$ ' 36.3" O. EPN 11676); [38] Wisui comunidad, Parroquia Macuma, Taisha, Morona Santiago (2 20' 24.7" S, 77² 27' 33.6" O. EPN 11949, 11950, 11967); [39] La Libertad, Parque Nacional Sangay, Méndez, Morona Santiago ( $2^{\circ} 43^{\prime} 53.2^{\prime \prime} \mathrm{S}, 78^{\circ} 18^{\prime} 35.5^{\prime \prime}$ O. EPN12176); [40] Parroquia Curaray, Cantón Araujuno, Pastaza ( ${ }^{\circ}$

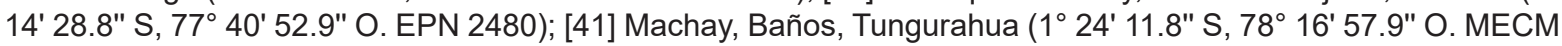
1); [42] Parque Nacional Sangay, Sinaí, Morona Santiago (1 $1^{\circ} 48^{\prime} 0^{\prime \prime} \mathrm{S}, 77^{\circ} 55^{\prime} 60^{\prime \prime} \mathrm{O}$. MECM 4103, 4196, 4242); [43] Bloque 27 (Putumayo), Cantón Santa Elena, Sucumbíos (0²2' 7.2" N, 76 11' 8.4" O. MECM3365, 3370). 